\title{
Beyond dynamic density functional theory: the role of inertia
}

\author{
Umberto Marini Bettolo Marconi ${ }^{1}$, Pedro Tarazona ${ }^{2}$, \\ Fabio Cecconi ${ }^{3}$ and Simone Melchionna ${ }^{4}$ \\ ${ }^{1}$ Dipartimento di Fisica, Via Madonna delle Carceri, I-68032 Camerino (MC), Italy \\ ${ }^{2}$ Departamento de Fisica Teórica de la Materia Condensada and Instituto Nicolás Cabrera, \\ Universidad Autonoma de Madrid, E-28049 Madrid, Spain \\ ${ }^{3}$ INFM-CNR Center for Statistical Mechanics and Complexity, and Institute for Complex \\ Systems CNR, Via dei Taurini 19, I-00182 Rome, Italy \\ ${ }^{4}$ INFM-CNR and Dipartimento di Fisica, Università La Sapienza, I-00185 Rome, Italy
}

E-mail: umberto.marinibettolo@unicam.it,pedro.tarazona@uam.es,

fabio.cecconi@roma1.infn.it and simone.melchionna@roma1.infn.it

Received 28 July 2008

Published 12 November 2008

Online at stacks.iop.org/JPhysCM/20/494233

\begin{abstract}
We discuss the recent attempts to generalize dynamical density functional (DDF) theory to situations where the momentum and energy transport, not necessarily associated with mass diffusion, play a role. We consider an assembly of particles described by inertial dynamics and subjected to the influence of a heat-bath. By means of a time multiple timescale analysis we derive the evolution equation for the noise-averaged density field. Remarkably, for large values of the friction parameter and/or the mass of the particles we obtain the same governing equation of DDF, and in addition we are able to compute higher-order corrections.
\end{abstract}

\section{Introduction}

A massive effort has been devoted, in recent years, to the study of non-uniform systems at thermodynamic equilibrium and firm theoretical approaches have been established. Among these, density functional theory (DFT) [1] is perhaps the most versatile. In contrast, we do not have a similar control over the behavior of systems in generic non-equilibrium situations. However, if the damped character of the dynamics makes the density the only relevant field, dynamical extensions of DFT have been introduced and tested successfully, as for the important case of colloidal suspensions.

In the more general case, one needs to fully account for the momentum and energy transport in addition to the dynamics of the density field. The natural bottom-up approach is to consider the evolution of both positions and momenta of the particles and derive the governing equation for the phase-space distribution, conveying the information about the microscopic nature of the fluid. In this work we consider a system of interacting particles subjected to thermostatted inertial dynamics and derive the governing time-dependent equation for the one-body density [2]. We show that, after a suitable truncation of the Bogoliubov-Born-Green-Kirkwood-Yvon hierarchy and a multiple timescale analysis, we obtain a self-consistent equation involving only the one-body density distribution [3]. Among the advantages of the present approach over the standard time-dependent density functional method are: (a) the possibility to treat assemblies of inelastic particles [4], i.e. systems with interactions which do not conserve the energy, (b) the ability to deal with non-isothermal systems and (c) a better description of systems in the presence of time-dependent potentials. As an application of the method, we illustrate some results concerning the inhomogeneous properties of inelastic hard rods in the presence of an external potential. The present theory has also been extended to arbitrary dimensions [5] and represents a route to derive transport coefficients.

\section{Theory}

We consider an assembly of $N$ particles moving in a $d$ dimensional region with positions $\mathbf{x}_{i}$ and velocities $\mathbf{u}_{i}(i=$ $1, N)$. The particles have the same mass, $m$, experience an external force $\mathbf{f}_{\mathrm{e}}(\mathbf{x})$ and interact through a pair potential $U\left(\left|\mathbf{x}-\mathbf{x}^{\prime}\right|\right)$. The model also assumes that the particles are subjected to a viscous drag force proportional to their velocity plus a random stochastic forcing, arising from a heat-bath. The 
equations of motion are

$$
\begin{aligned}
& \mathrm{d} \mathbf{x}_{n}=\mathbf{u}_{n} \mathrm{~d} t \\
& m \mathrm{~d} \mathbf{u}_{n}=\left[\mathbf{f}_{\mathrm{e}}\left(\mathbf{x}_{n}\right)-\sum_{m(\neq n)} \nabla_{\mathbf{r}_{n}} U\left(\left|\mathbf{x}_{n}-\mathbf{x}_{m}\right|\right)-m \gamma \mathbf{u}_{n}\right] \mathrm{d} t \\
& \quad+\mathrm{d} \mathbf{W}_{n}(t)
\end{aligned}
$$

where $\mathrm{d} \mathbf{W}_{n}(t)=\boldsymbol{\xi}_{n}(t) \mathrm{d} t$ is the increment of the Wiener process and $\xi_{n}(t)$ is a Gaussian white noise with properties

$$
\begin{gathered}
\left\langle\xi_{n}^{i}(t)\right\rangle=0 \\
\left\langle\xi_{n}^{i}(t) \xi_{m}^{j}(s)\right\rangle=2 \gamma m k_{\mathrm{B}} T \delta_{m n} \delta^{i j} \delta(t-s)
\end{gathered}
$$

where $T$ is the 'heat-bath temperature' and $\langle\cdot\rangle$ indicates the average over a statistical ensemble of noise realizations [6].

By a standard procedure of stochastic calculus [7, 8] we rewrite the description based on the trajectories into a description based on the $N$-body phase-space distribution, $f^{(N)}(\{\mathbf{x}, \mathbf{v}\}, t)$ :

$$
\begin{aligned}
\left(\frac{\partial}{\partial t}\right. & +\sum_{n}\left[\mathbf{v}_{n} \cdot \nabla_{\mathbf{x}_{n}}+\frac{\mathbf{f}_{\mathbf{e}}\left(\mathbf{x}_{n}\right)}{m} \cdot \nabla_{\mathbf{v}_{n}}\right. \\
& \left.\left.-\gamma\left[\nabla_{\mathbf{v}_{n}} \cdot \mathbf{v}_{n}+\frac{k_{\mathrm{B}} T}{m} \nabla_{\mathbf{v}_{n}} \cdot \nabla_{\mathbf{v}_{n}}\right]\right]\right) f^{(N)}(\{\mathbf{x}, \mathbf{v}\}, t) \\
= & \frac{1}{m} \sum_{n} \sum_{m(\neq n)} \nabla_{\mathbf{x}_{n}} U\left(\left|\mathbf{x}_{n}-\mathbf{x}_{m}\right|\right) \cdot \nabla_{\mathbf{v}_{n}} f^{(N)}(\{\mathbf{x}, \mathbf{v}\}, t) .
\end{aligned}
$$

In practice, only the information contained in the first two reduced distribution functions, $f^{(1)}$ and $f^{(2)}$, obtained from $f^{(N)}$ by integrating over the phase-space coordinates of $(N-1)$ and $(N-2)$ particles, respectively, is relevant to our study. The equation for $f^{(1)}$ is

$$
\begin{aligned}
& \frac{\partial}{\partial t} f^{(1)}(\mathbf{x}, \mathbf{v}, t)+\left[\mathbf{v} \cdot \nabla_{\mathbf{x}}+\frac{\mathbf{f}_{\mathrm{e}}(\mathbf{x})}{m} \cdot \nabla_{\mathbf{v}}\right] f^{(1)}(\mathbf{x}, \mathbf{v}, t) \\
& \quad=\gamma\left[\nabla_{\mathbf{v}} \cdot \mathbf{v}+\frac{k_{\mathrm{B}} T}{m} \nabla_{\mathbf{v}} \cdot \nabla_{\mathbf{v}}\right] L_{\mathrm{FP}} f^{(1)}(\mathbf{x}, \mathbf{v}, t)+k(\mathbf{x}, \mathbf{v}, t)
\end{aligned}
$$

where the left-hand side of equation (5) contains the free streaming of the particles, while the right-hand side accounts for the interaction with the heat-bath and among particles [9]. The interaction term $k(\mathbf{x}, \mathbf{v}, t)$, in the case of continuous potentials, is

$$
\begin{gathered}
k(\mathbf{x}, \mathbf{v}, t)=\frac{1}{m} \nabla_{\mathbf{v}} \int \mathrm{d} \mathbf{x}^{\prime} \int \mathrm{d} \mathbf{v}^{\prime} f^{(2)}\left(\mathbf{x}, \mathbf{v}, \mathbf{x}^{\prime}, \mathbf{v}^{\prime}, t\right) \\
\times \nabla_{\mathbf{x}} U\left(\left|\mathbf{x}-\mathbf{x}^{\prime}\right|\right)
\end{gathered}
$$

and depends on the two-particle distribution function, $f^{(2)}\left(\mathbf{x}, \mathbf{v}, \mathbf{x}^{\prime}, \mathbf{v}^{\prime}, t\right)$, so that an approximate closure is required in order to truncate the hierarchy. We first approximate the doublet distribution function as $f^{(2)}\left(\mathbf{x}, \mathbf{x}^{\prime}, \mathbf{v}, \mathbf{v}^{\prime}, \tau\right)=$ $f^{(1)}(\mathbf{x}, \mathbf{v}, \tau) f^{(1)}\left(\mathbf{x}^{\prime}, \mathbf{v}^{\prime}, \tau\right) g_{2}\left(\mathbf{x}, \mathbf{x}^{\prime} \mid \rho\right)$ in which the pair correlation $g_{2}$ is a function only of the particle positions, but not of their velocities. It is assumed that $g_{2}$ is a non-local equilibrium functional of the profile $\rho(\mathbf{x}, t)$ and depends on time only through the density, and has the same form as in a nonuniform equilibrium state whose density is $\rho(\mathbf{x}, t)$. With this approximation we find

$$
k(\mathbf{x}, \mathbf{v}, \tau)=-\mathbf{F}^{\mathrm{mol}}(\mathbf{x}, \tau) \cdot \nabla_{\mathbf{v}} f^{(1)}(\mathbf{x}, \mathbf{v}, \tau)
$$

where, using $\rho(\mathbf{x}, \tau) \sigma^{d}=\int \mathrm{d} \mathbf{v} P(\mathbf{x}, \mathbf{v}, \tau)$, we have introduced the molecular field:

$$
\mathbf{F}^{\mathrm{mol}}(\mathbf{x}, \tau)=-\int \mathrm{d} \mathbf{x}^{\prime} \rho\left(\mathbf{x}^{\prime}, \tau\right) g_{2}\left(\mathbf{x}, \mathbf{x}^{\prime} \mid \rho\right) \nabla_{\mathbf{x}} U\left(\left|\mathbf{x}-\mathbf{x}^{\prime}\right|\right) .
$$

For particles with hard-core interactions the term $\nabla_{\mathbf{x}} U\left(\left|\mathbf{x}-\mathbf{x}^{\prime}\right|\right)$ is ill-defined and the mean-field treatment is not appropriate, since the force has an impulsive character. Due to the hard-core interaction the velocities change instantaneously and their value after the impact, denoted with a prime, is related to the one before the impact (unprimed) by the linear transformation:

$$
\begin{aligned}
& \mathbf{v}_{1}^{\prime}=\mathbf{v}_{1}-\left(\mathbf{v}_{12} \cdot \hat{\boldsymbol{\sigma}}\right) \hat{\boldsymbol{\sigma}} \\
& \mathbf{v}_{2}^{\prime}=\mathbf{v}_{2}+\left(\mathbf{v}_{12} \cdot \hat{\boldsymbol{\sigma}}\right) \hat{\boldsymbol{\sigma}}
\end{aligned}
$$

where $\mathbf{v}_{12}=\mathbf{v}_{1}-\mathbf{v}_{2}$ and $\hat{\sigma}$ is the unit vector directed from particle 1 to particle 2. The revised Enskog theory (RET) [10, 11] provides a closed approximate expression for the collision term as a function of the single-particle distribution function:

$$
\begin{aligned}
& k\left(\mathbf{x}_{1}, \mathbf{v}_{1}, t\right)=\sigma^{d-1} \int \mathrm{d} \mathbf{v}_{2} \int \mathrm{d} \hat{\boldsymbol{\sigma}} \Theta\left(\hat{\boldsymbol{\sigma}} \cdot \mathbf{v}_{12}\right)\left(\hat{\boldsymbol{\sigma}} \cdot \mathbf{v}_{12}\right) \\
& \quad \times\left\{g_{2}\left(\mathbf{x}_{1}, \mathbf{x}_{1}-\hat{\boldsymbol{\sigma}} \mid \rho\right) f^{(1)}\left(\mathbf{x}_{1}, \mathbf{v}_{1}^{\prime}, \tau\right) f^{(1)}\left(\mathbf{x}_{1}-\hat{\boldsymbol{\sigma}}, \mathbf{v}_{2}^{\prime}, \tau\right)\right. \\
& \left.-g_{2}\left(\mathbf{x}_{1}, \mathbf{x}_{1}+\hat{\boldsymbol{\sigma}} \mid \rho\right) f^{(1)}\left(\mathbf{x}_{1}, \mathbf{v}_{1}, \tau\right) f^{(1)}\left(\mathbf{x}_{1}+\hat{\boldsymbol{\sigma}}, \mathbf{v}_{2}, \tau\right)\right\}
\end{aligned}
$$

where the primes on the velocities denote scattered values determined from equation (9). It is important to recognize that pairs of atoms are assumed to be uncorrelated immediately prior to collision but are correlated after they collide, i.e. collision itself generates correlations.

It is convenient to switch to non-dimensional variables which are obtained by measuring the velocities in units of the thermal velocity $v_{\mathrm{T}}=\sqrt{k_{\mathrm{B}} T / m}$ and lengths in units of $\sigma$, i.e. $\mathbf{V} \equiv \frac{\mathbf{v}}{v_{\mathrm{T}}}$ and $\mathbf{X} \equiv \frac{\mathbf{x}}{\sigma}$. The remaining variables can be non-dimensionalized according to the transformations $\tau \equiv t \frac{v_{\mathrm{T}}}{\sigma}, \Gamma \equiv \gamma \frac{\sigma}{v_{\mathrm{T}}}, \mathbf{F}(\mathbf{X}) \equiv \frac{\sigma}{m v_{\mathrm{T}}^{2}} \mathbf{f}_{e}(\mathbf{x})$. Finally, the distribution function and the collision term are rescaled according to the transformations: $P(\mathbf{X}, \mathbf{V}, \tau) \equiv \sigma^{d} v_{\mathrm{T}}^{d} f^{(1)}(\mathbf{x}, \mathbf{v}, t)$ and $K(\mathbf{X}, \mathbf{V}, \tau) \equiv \sigma^{d+1} v_{\mathrm{T}}^{d-1} k(\mathbf{x}, \mathbf{v}, t)$, where $d$ is the dimensionality of the embedding space.

For the sake of simplicity, hereafter we shall specialize to one dimension where the notation becomes much simpler. Equation (5) can be cast in the following non-dimensional form:

$$
\begin{aligned}
& \frac{1}{\Gamma} \frac{\partial P(X, V, \tau)}{\partial \tau}=\left[L_{\mathrm{FP}}-\frac{1}{\Gamma} V \frac{\partial}{\partial X}-\frac{1}{\Gamma} F(X, \tau) \frac{\partial}{\partial V}\right] \\
& \times P(X, V, \tau)+\frac{1}{\Gamma} K(X, V, \tau)
\end{aligned}
$$

having introduced for the sake of brevity the 'Fokker-Planck' operator $L_{\mathrm{FP}}$ whose eigenfunctions $H_{\mu}(V)$ have the property

$$
L_{\mathrm{FP}} H_{\mu}(V) \equiv \frac{\partial}{\partial V}\left[\frac{\partial}{\partial V}+V\right] H_{\mu}(V)=-\mu H_{\mu}(V),
$$

for $\mu=0,1, \ldots$, and have the explicit representation

$$
H_{\mu}(V) \equiv \frac{1}{\sqrt{2 \pi}}(-1)^{\mu} \frac{\partial^{\mu}}{\partial V^{\mu}} \exp \left(-\frac{1}{2} V^{2}\right) .
$$


It is also convenient to look for solutions of equation (12) where the position and velocity dependence of the distribution function can be separated by projecting onto the basis of the eigenfunctions $H_{\mu}(V)$. To this purpose we define raising and lowering operators, $a_{ \pm} H_{\mu}(V)=H_{\mu \pm 1}(V)$, respectively, with the properties

$$
V H_{\mu}(V)=\left(a_{+}+\mu a_{-}\right) H_{\mu}(V),
$$

and expand over the basis set $H_{v}(V)$, both the phase-space distribution

$$
P(X, V, \tau)=\sum_{v} \Phi_{v}(X, \tau) H_{v}(V)
$$

and the collision term

$$
K(X, V, \tau)=\sum_{v} C_{v}(X, \tau) H_{v}(V) .
$$

As shown in our previous work, the coefficients $C_{v}(X, \tau)$ have simple expressions in terms of the moments $\Phi_{v}(X, \tau)$ with coefficients which are non-local functionals of the density distribution. Substituting equations (15) and (16) in equation (11) and using the orthogonality of the basis set $H_{v}(V)$, we obtain a system of coupled equations for the moments $\Phi_{v}(X, \tau)$ which can be written in compact form as

$$
\begin{aligned}
& {\left[\frac{\partial}{\partial \tau}+\Gamma v\right] \Phi_{\nu}(X, \tau)+(v+1) \frac{\partial \Phi_{v+1}(X, \tau)}{\partial X}} \\
& \quad+\left[\frac{\partial}{\partial X}-F(X)\right] \Phi_{\nu-1}(X, \tau)-C_{\nu}(X, \tau)=0
\end{aligned}
$$

with $\Phi_{-1}=0$. We identify the moment $\Phi_{0}(X, \tau)=$ $\int \mathrm{d} V P(X, V, \tau)$ with the number density, $\rho(X, \tau), \Phi_{1}(X, \tau)=$ $\int \mathrm{d} V V P(X, V, \tau)$ with the momentum density $J(X, \tau)$ or particle current, and $\Phi_{2}(X, \tau)+\Phi_{0}(X, \tau) / 2=$ $1 / 2 \int \mathrm{d} V V^{2} P(X, V, \tau)$ with the kinetic energy density.

Before trying to solve the full problem, it is somehow instructive to consider the exact solutions of equation (11) for a system of free particles $(F=0$ and $K=0)$. These can be written in terms of the infinite series of modes, $\mu=0,1, \ldots$, with the generic form [3]

$$
\begin{gathered}
P^{(\mu)}(X, V, \tau)=\exp (-\mu \Gamma \tau) \exp \left[-\frac{a_{+}}{\Gamma} \frac{\partial}{\partial X}\right] \\
\times\left(1+\frac{a_{-}}{\Gamma} \frac{\partial}{\partial X}\right)^{\mu} H_{\mu}(V) \phi^{(\mu)}(X, \tau) .
\end{gathered}
$$

The function $\phi^{(\mu)}(X, \tau)$, which fully defines the mode $\tilde{P}^{(\mu)}(X, V, \tau)$, represents any solution of the diffusion equation

$$
\frac{\partial}{\partial \tau} \phi^{(\mu)}(X, \tau)=\frac{1}{\Gamma} \frac{\partial^{2}}{\partial X^{2}} \phi^{(\mu)}(X, \tau)
$$

Clearly, the solutions with $\mu>0$ decay fast and the only relevant contribution to the phase-space distribution is represented by the $\mu=0$ mode. The dominant contribution to this mode is a density fluctuation (the first term), followed by a current fluctuation (the term of order $\Gamma^{-1}$ ) and so on according to the structure

$$
\begin{aligned}
& P^{(0)}(X, V, \tau)=H_{0}(V) \phi^{(0)}(X, \tau)-\frac{H_{1}(V)}{\Gamma} \frac{\partial \phi^{(0)}(X, \tau)}{\partial X} \\
& +\frac{H_{2}(V)}{2 ! \Gamma^{2}} \frac{\partial^{2} \phi^{(0)}(X, \tau)}{\partial X^{2}}+\cdots
\end{aligned}
$$

All terms are slaved by the first and their shapes are given by the successive derivatives of $\phi^{(0)}(X, \tau)$ with respect to $X$. Indeed, the amplitudes of the $H_{1}(V)$ and $H_{2}(V)$ components are completely determined from knowledge of $\phi^{(0)}(X, \tau)$. Such a complexity reduction occurs because the density is the only conserved field in our thermostatted model [12].

\section{Multiple timescale method}

As is clear from the previous illustrative discussion, what really matters is the evolution of the density field which occurs slowly by diffusion. In turn, such a field generates a momentum current, an energy current and so on through the spatial derivatives of increasing order. In the case of non-interacting particles, these currents do not affect the evolution of the density, whereas they induce a feedback in the interacting case. In the fully interacting case, the problem of deriving from equation (5) the correct form of the governing equation for the density can be solved by means of the multiple timescale analysis [13, 14]. In a nutshell, the method makes use of the presence of two distinct timescales. The first scale is fast and corresponds to the time interval necessary for the velocities to relax to configurations consistent with their thermal equilibrium value. The second timescale is much longer and corresponds to the time necessary for the positions of the particles to assume their equilibrium configurations. We omit here the lengthy analytical derivation, which has already been presented elsewhere [3], and write only the final result:

$$
\frac{\partial \rho}{\partial \tau}(X, \tau)=\frac{1}{\Gamma} \partial_{X}\left\{\left[J_{\mathrm{DFT}}(X, \tau)+\delta J(X, \tau)\right\} .\right.
$$

constituting a closed expression for the density $\rho(X, \tau)$. As shown below, the density field fully characterizes the state of the system and slaves the remaining hydrodynamic fields. The leading term of order $\Gamma^{-1}$ can be written as

$$
J_{\mathrm{DFT}}(X, \tau)=-\rho(X, \tau) \partial_{X}\left[\frac{\delta F^{\mathrm{rod}}[\rho]}{\delta \rho(X, \tau)}+V_{\mathrm{ext}}(X)\right],
$$

where $F^{\mathrm{rod}}[\rho]$ is the hard-rod density functional of the instantaneous ensemble averaged density $\rho(X, \tau)$ and the label DFT indicates that this contribution to the particle current is identical to the contribution predicted by DDFT.

The second term in the rhs of order $\Gamma^{-2}$ is

$$
\begin{aligned}
& \delta J(X, \tau)=\frac{2}{\sqrt{\pi} \Gamma} \rho(X, \tau)\left[g_{2}(X, X+1) J_{\mathrm{DFT}}(X+1, \tau)\right. \\
& \left.\quad+g_{2}(X, X-1) J_{\mathrm{DFT}}(X-1, \tau)\right] \\
& \quad-\frac{2}{\sqrt{\pi}} J_{\mathrm{DFT}}(X, \tau)\left[g_{2}(X, X+1) \rho(X+1, \tau)\right. \\
& \left.\quad+g_{2}(X, X-1) \rho(X-1, \tau)\right]
\end{aligned}
$$


and is a correction to the DDFT result accounting for the departures of the velocity distribution from the Maxwellian shape, which are not considered in DDFT approaches. The current is a functional of the scaled density $\rho(X, \tau)$, so that equation (21) is self-consistent and can be solved numerically by iteration. It is important to say that, to establish this equation, we have not used the character of the free energy or other equilibrium considerations, but only the form of the pair correlation function which enters the RET closure. We believe that this result is remarkable because it establishes that the present kinetic derivation leads to the same DDFT equation if the inverse friction expansion is truncated at the lowest order. On the other hand, when higher-order corrections are included we were unable to prove an $\mathrm{H}$-theorem stating that there exists a Lyapunov functional of the dynamics which is a non-increasing function of time.

\section{Granular systems}

Granular gases, i.e. assemblies of particles experiencing mutual energy non-conserving collisions, are often represented by means of inelastic hard spheres [15]. Inelastic hard spheres in one dimension (hard rods) obey the following collision rule, whenever the separation of two particles, say $i$ and $i+1$, equals the hard-rod length $\sigma$ :

$$
\begin{aligned}
& v_{1}^{\prime}=v_{1}+\frac{1+\alpha}{2 \alpha}\left(v_{2}-v_{1}\right) \\
& v_{2}^{\prime}=v_{2}-\frac{1+\alpha}{2 \alpha}\left(v_{2}-v_{1}\right) .
\end{aligned}
$$

where the precollisional velocities are indicated with primed symbols and the postcollisional velocities are unprimed. The collision manifestly conserves the total momentum of the pair, but not the total kinetic energy. The granular gas is therefore bound to cool and eventually come to rest, since its kinetic temperature, proportional to the average kinetic energy per particle, decreases as collisions take place, unless a sufficient amount of energy is injected into the system to balance the inelastic losses. A simple way to achieve a steady state regime is to couple the inelastic particles to an energy source idealized as a heat-bath. Such an assumption renders the model somehow simpler and allows us to use the same methods employed to study colloidal systems, but with a collision kernel modified by inelasticity. We give here only the final result of the analytical treatment, leading to the equation for the density analogous to equation (21). The new feature which comes about is the presence of a new contribution to the particle current:

$\delta J^{\prime}(X, \tau)=-\partial_{X} \frac{\left(1-\alpha^{2}\right)}{2 \sqrt{\pi} \Gamma} \rho(X, \tau)\left[g_{2}(X, X+1) \rho(X+1, \tau)\right.$
$\left.\quad+g_{2}(X, X-1) \rho(X-1, \tau)\right]$ that vanishes in the limit $\alpha \rightarrow 0$ and for uniform density distribution, and describes the tendency of the particles to form locally dense aggregates due to their inelasticity [4].

Not too surprisingly, in the light of the previous analysis of colloidal systems, this is an equation involving the density field alone, but the presence of extra collisional terms signals the coupling between density fluctuations and temperature fluctuations. The latter in turn are slaved by the density fluctuations so that the equation is self-consistent.

In the limit $\tau \rightarrow \infty$ we can determine the granular temperature profile:

$$
\begin{aligned}
& T(X)=1-\frac{\left(1-\alpha^{2}\right)}{2 \Gamma \sqrt{\pi}}\left[g_{2}(X, X+1) \rho(X+1)\right. \\
& \left.\quad+g_{2}(X, X-1) \rho(X-1)\right]
\end{aligned}
$$

where we suppressed the time argument and the functions of the single spatial argument have to be understood as their asymptotic limiting values when $\tau \rightarrow \infty$. The constant 1 in the rhs represents (in our reduced units) the heat-bath temperature, whereas the second term is the shift in the local temperature induced by collisions. In fact, it amounts to the product of three factors: the kinetic energy dissipated, the collision rate $\omega_{\mathrm{E}}$ (Enskog collision frequency) and the typical time $\gamma^{-1}$ of the heat-bath. The average Enskog frequency at each side of the particle located at $X$ is

$$
\frac{\omega_{\mathrm{E}}(X \pm 1)}{\gamma}=\frac{2}{\Gamma \sqrt{\pi}} g_{2}(X, X \pm 1) \rho(X \pm 1)
$$

and in the case of a uniform system it reduces to the bulk Enskog frequency $\omega_{\mathrm{E}}=2 v_{\mathrm{T}} \rho \sigma g_{2} / \sqrt{\pi}$, where $v_{\mathrm{T}}$ is the thermal velocity of the gas.

We turn now our attention to the pressure profile $\Pi(X, \tau)$, which can be separated into a kinetic and a collisional contribution:

$$
\Pi(X)=\Pi_{\mathrm{kin}}(X)+\Pi_{\mathrm{coll}}(X) .
$$

The first term is the so-called kinetic pressure, arising from the momentum transfer associated with the particle motion:

$$
\Pi_{\text {kin }}(X)=T(X) \rho(X) .
$$

In the same limit, taking into account the collisional pressure, i.e. the contribution stemming from the momentum transfer through collisions acting even in the absence of mass transport, one finds the equation of state in the uniform non-equilibrium steady state in the form

$$
\Pi=T \rho\left(1+\frac{(1+\alpha)}{2} \frac{\rho}{1-\rho}\right) .
$$

Expressing the temperature as a function of the density (from equation (26))

$$
T=1-\frac{1-\alpha^{2}}{\Gamma \sqrt{\pi}} g_{2} \rho
$$

we see that equation (30) describes the lowering of the pressure due to the collisional reduction of the temperature and becomes the familiar hard-rod pressure equation for $\alpha=1$.

\section{Systems with non-uniform temperature}

Thermal gradients, besides causing a heat flow, can induce a mass flow in systems such as colloids. The phenomenon is termed thermodiffusion, thermophoresis or the LudwigSoret effect $[16,17]$ and is relevant because it represents 
a tool to manipulate and concentrate molecules in solution. Thermophoresis, in fact, typically enhances the concentration in colder regions.

Nevertheless, in spite of its technological interest, not many studies provide a derivation of the governing equation for the concentration field in the presence of a temperature gradient externally imposed (see, however, [18]). A problem arises when one considers the $\gamma \rightarrow \infty$ limit: the resulting stochastic equation contains a multiplicative noise term (the temperature is space-dependent). This in turn means that the associated FPE depends on the particular prescription used to derive it, i.e. one encounters the so-called Ito-Stratonovich dilemma. To solve it, Matsuo and Sasa [19] recently obtained the Smoluchowski [20] equation from a perturbation expansion in powers of the inverse friction. Two important aspects arise from their work: first, the underdamped dynamics is free from the Ito-Stratonovich dilemma and, second, their perturbation scheme is unbounded in time and one needs a renormalization treatment to make it convergent.

Even in this case the multiple timescale method has been employed to derive the Smoluchowski equation for a system of Brownian particles in a temperature gradient derived from the Kramers' equation [21]. Following the line of reasoning of Marconi and Tarazona [3], one could write the following governing equation:

$$
\begin{aligned}
& \frac{\partial \rho(x, t)}{\partial t}=\nabla\{\nabla D(x) \rho(x, t) \\
& \left.\quad+\frac{1}{m \gamma} \rho(x, t) \nabla\left[\frac{\delta \mathcal{F}_{\text {ni }}[\rho]}{\delta \rho(x, t)}+V_{\text {ext }}(x)\right]\right\},
\end{aligned}
$$

where $D(x)=k_{\mathrm{B}} T(x) / m \gamma$ and $\mathcal{F}_{\text {ni }}$ is the excess over the ideal gas contribution to the free energy. The second term renormalizes the effective diffusion constant of the particles and may become important at higher concentrations.

\section{Final remarks}

We have considered the non-equilibrium dynamics of a colloidal system of mass $m$ subjected to a uniform heatbath. The evolution depends on the non-dimensional frictional parameter $\Gamma^{-1}$, proportional to the time span occurring for the velocity distribution to reach its equilibrium value, and the packing fraction. This evolution is described by a Kramers' equation for the phase-space distribution supplemented by a collision term, treated within the revised Enskog theory. Since the momentum degrees of freedom equilibrate much faster than the positional degrees of freedom, it is reasonable to look for a description which contains only the latter variables. By employing a multiple timescale analysis we obtained a modified Smoluchowski-Enskog equation for the density field and found that the collision term gives a non-local coupling between density, momentum and energy fluctuations. However, the density field slaves the remaining fields. To lowest order in $\Gamma^{-1}$ the present method yields the same evolution equation for the density as the one obtained within the DDF approach. The present derivation does not require the existence of any equilibrium density functional, but is based on kinetic theory arguments. Therefore, it can be applied to generic non-equilibrium systems, where the RET closure is physically sound. However, by containing as a key ingredient the same equilibrium pair correlation as the DDF, the matching between the two methods is not too surprising.

As discussed by Archer and Evans [22] if the thermal equilibration occurs mainly via the solvent the deviations from the DDF should be negligible. Nevertheless, for atomic fluids the harshly repulsive potential might concur appreciably to the relaxation process and lead to significant effects which are beyond the limits of the DDF approach. In this respect, notice that the proposal made by Archer [23] for a DDF approximation for the fully inertial case was based on the direct use of the molecular field equation (8). Our analysis here shows the importance of going beyond that approximation even at the first correction from the fully damped limit, since the hard-core repulsions create a strong correlation between the relative velocities of the colliding particles.

Besides reproducing known results the present derivation provides systematic corrections to the DDF equation accounting for the deviation of the velocity distribution from the Maxwellian. Hence, it can describe situations very far from thermodynamic equilibrium or even situations where a steady non-equilibrium state exists.

The present method quite naturally lends itself to the following future applications and extensions: (a) systems of particles experiencing inelastic collisions, such as granular gases, where free energy functional approaches are not applicable and the RET closure provides a valid alternative, (b) systems having a non-uniform temperature profile where the standard isothermal DDF approach cannot be applied and (c) inclusion of higher-order corrections in the inverse friction expansion $\Gamma^{-1}$ accounting for currents associated with higher moments of the velocity distribution.

\section{Acknowledgments}

PT acknowledges the Direccion General de investigacion of Spain (grant FIS2007-65869-C03-01) and the Comunidad Autonomoma de Madrid (grant s-0505/ESP-0299).

\section{References}

[1] Evans R 1979 Adv. Phys. 28143

[2] Marini Bettolo Marconi U and Tarazona P 1999 J. Chem. Phys. 1108032

Marini Bettolo Marconi U and Tarazona P 2000 J. Phys.: Condens. Matter 12413

[3] Marini Bettolo Marconi U and Tarazona P 2006 J. Chem. Phys. 124164901

[4] Marini Bettolo Marconi U, Tarazona P and Cecconi F 2007 J. Chem. Phys. 126164904

[5] Marini Bettolo Marconi U and Melchionna S 2007 J. Chem. Phys. 126184109

[6] Cecconi F, Puglisi A, Marini Bettolo Marconi U and Vulpiani A 2003 Phys. Rev. Lett. 90064301

[7] Gardiner C 1994 Handbook of Stochastic Methods for Physics, Chemistry and in the Natural Sciences (Berlin: Springer)

[8] Risken H 1984 The Fokker-Planck Equation (Berlin: Springer)

[9] Kramers H A 1940 Physica A 7284

[10] van Beijeren H and Ernst M H 1973 Physica 68437 van Beijeren H and Ernst M H 1973 Physica 70225 
[11] Santos A, Montanero J M, Dufty J W and Brey J J 1998 Phys. Rev. E 571644

Dufty J W, Santos A and Brey J 1996 Phys. Rev. Lett. 771270

[12] Tarazona P and Marini Bettolo Marconi U 2008 J. Chem. Phys. 128164704

[13] Wilemski G 1976 J. Stat. Phys. 14153

[14] Bocquet L 1997 Am. J. Phys. 65140

[15] Cecconi F, Diotallevi F, Marini Bettolo Marconi U and Puglisi A 2004 J. Chem. Phys. 12035

Cecconi F, Diotallevi F, Marini Bettolo Marconi U and Puglisi A 2004 J. Chem. Phys. 1215125
[16] Landau L and Lifshitz E 1951 Fluid Mechanics (Moscow: Mir)

[17] de Groot S R and Mazur P 1984 Non-equilibrium Thermodynamics (New York: Dover)

[18] van Kampen N G 1988 J. Phys. Chem. Solids 49673

[19] Matsuo M and Sasa S-I 2000 Physica A 276188

[20] von Smoluchowski M 1916 Ann. Phys. 481103

[21] Lopez C and Marini Bettolo Marconi U 2007 Phys. Rev. E 75021101

[22] Archer A J and Evans R 2004 J. Chem. Phys. 1214246

[23] Archer A J 2005 J. Phys.: Condens. Matter 171405 